Saudi Journal of Biomedical Research

Abbreviated Key Title: Saudi J Biomed Res ISSN 2518-3214 (Print) |ISSN 2518-3222 (Online)

\title{
Assessment of Liver Function Change in Epileptic Patient Use Antiepileptic Drugs in Khartoum State (2019)
}

\author{
Reeham Abdalhamed Osman ${ }^{1 *}$ and Siddig Bushra Mohamed ${ }^{2}$ \\ ${ }^{1}$ Department of clinical chemistry, Faculty of medical laboratory sciences, University of Alneelain, Khartoum, Sudan \\ ${ }^{2}$ Department of clinical chemistry, Medical laboratory sciences program, Academy of Health Sciences, Khartoum, Sudan
}

DOI: $10.36348 /$ sjbr.2021.v06i03.001 $\quad$ | Received: 12.02.2021 | Accepted: 19.03.2021 | Published: 28.03 .2021

*Corresponding author: Reeham Abdalhamed Osman

\section{Abstract}

Background and aim: There are limited and confidante data about the effect of antiepileptic drugs in liver function. The aim of this study is to assess of carbamazepine, sodium valproate and phenytoin on plasma liver enzymes in epileptic Sudanese patients. Methods: This is an analytical case control study, conducted at National Center for Neurological Diseases and Sciences, Omdurman, Bahri and Academic Teaching Hospitals. A total of 64 epileptic patients were categorized into two groups, group I epileptic patients on antiepileptic drugs for more than one years and group II newly diagnosed epileptic patients and not started antiepileptic drugs. Analysis of AST, ALT, ALP activate and TP, Alb concentration was done by full automated machine. Results: The study showed that among group (I) there was significant increase in mean serum concentration AST 41.4 \pm 33.7 , ALT 24.8 \pm 12.65 with (p-value 0.975 and 0.016 ) respectively, compared with mean values of control (II) who reported AST 41.2 \pm 20.5 , ALT 16.8 \pm 9.66 . There was significant decrease in mean plasma concentration of ALP (62.4 \pm 39.9$)$ in Group (I) compared with mean values in group (II) (76.1 \pm 32.5 ) (p-value 0.137). Significant decrease in mean plasma albumin concentration $3.89 \pm 1.01$ in group (I) in contrast to group (2) $4.55 \pm 1.05$ (p-value 0.014 ). No significant change in total protein $7.36 \pm 1.08$, total bilirubin $0.91 \pm 0.84$, direct bilirubin $0.17 \pm 0.09$, between the two groups (T.B0.63 \pm 0.49$)$, (D.B0.18 \pm 0.12$)$. Conclusion: The study revealed that antiepileptic drugs had significant effects in increasing liver enzymes and this necessitate continuous screening of liver function and do of drug monitoring test among epileptic patients to overcome the adverse effects of these drugs on liver.

Keywords: PHT Phenytoin, VPA Valproic acid, CBZ carbamazepine, AST, ALT, ALP.

Copyright () 2021 The Author(s): This is an open-access article distributed under the terms of the Creative Commons Attribution 4.0 International License (CC BY-NC 4.0) which permits unrestricted use, distribution, and reproduction in any medium for non-commercial use provided the original author and source are credited.

\section{INTRODUCTION}

Epilepsy was defined conceptually in 2005 as a disorder of the brain characterized by an enduring predisposition to generate epileptic seizures. This definition is usually practically applied as having two unprovoked seizures $>24 \mathrm{~h}$ apart [1]. It is one of the leading neurological disorders worldwide affecting about 50 million people, and two-thirds of them are children [1-3]. Epilepsy is considered to be quite disabling because of its unpredictability $[4,5]$.

Antiepileptic drugs (AEDs) are no longer restricted to the treatment of epilepsy. These are widely used in a broad spectrum of psychiatric and neurological disorders. Liver plays a major role in the metabolism of a majority of these drugs. Hepatotoxicity is rare, but a real concern when initiating therapy.
Likewise, liver disease can adversely affect the biotransformation of some of these drugs [6].

Drug-induced liver injury associated with antiepileptic drugs (AED) is well recognized. The frequency of the most common AED is rare but the consequences can be very serious leading to death or liver transplantation due to acute liver failure induced by these drugs [7]. It is clear from above mentioned literature, that antiepileptic medications may affects the liver function of the patients, no previous studies touched this issue in our settings, accordingly this study assessed the effects of carbamazepine, sodium valproate and phenytoin on plasma liver enzymes in epileptic Sudanese patients. 


\section{MATERIALS AND METHODS}

Study design analytical case control study

\section{Study area}

The study was conducted at National Center for Neurological Diseases and Sciences, Omdurman, Bahri and Academic Teaching Hospitals in Khartoum State, Sudan.

\section{Study period}

The study was conducted during the period from June 2019 to August 2020.

\section{Study population}

The study population included epileptic patients at the above mentioned hospitals during the study period.

\section{INCLUSION CRITERIA}

Cases included adult Sudanese males and female diagnosed as epileptic patients based on EEG and use antiepileptic drugs for more than one years. Epileptic patients who received one of the following antiepileptic drugs (carbamazepine, sodium valproate or phenytoin) from at least 1 year. Control included in the study were Sudanese males and females aged above 18 and attended the above mentioned hospitals and diagnosed with epilepsy by EEG but not on carbamazepine, sodium valproate or phenytoin treatment.

\section{EXCLUSION CRITERIA}

Epileptic patients using other drugs causing elevation of liver enzymes e.g. antibiotics, antirheumatic drug); patients consumed alcohol; Patient diagnosed with chronic disease (diabetes, hepatitis and cardiovascular disease,...); non Sudanese patient and women has been taken estrogen or progesterone therapy

\section{Sample size}

The sample size was calculated according to the know formula which is used to reach a certain desired margin of error in the results, to give a maximum of error $(0.05)$ with probability of $(a=0.05)$ as follow;

$$
\mathrm{N}=\mathrm{z} 2 \times \mathrm{p} \times \mathrm{q}
$$

d2

$\mathrm{N}=3.3124 \times 0.025 \times 0.975 \% 0.0025=32.2$

$\mathrm{Z}=$ the value in normal curve corresponding to level of confidence $95 \%=1.96$

$\mathrm{P}=$ probability prevalence in the community is (highest prevalence reported in

Khartoum $2.5 \%$ ) or 0.025

$\mathrm{Q}=(1-\mathrm{p})=1-0.025$

$\mathrm{D}=$ margin of error $=0,05$

Based on the above formula, a total of 64 epileptic patients fulfilled the inclusion criteria of the study were enrolled. The patients divided into two groups, group (I) cases included 32 diagnosed by EEG that have epilepsy and on antiepileptic drugs for at least 1years. Group (II) included 32 patients diagnosed by EEG that have epilepsy and not on antiepileptic drugs.

\section{STATISTICAL ANALYSIS}

The data was analyzed using statistical packages for social sciences (SPSS) (Version 25). T test used for calculation of mean values of liver enzymes and other study variables. ANOVA test was used to compare between the means within the study variables. $P$ value considered significant if $\leq 0.05$.

\section{ETHICAL CONSIDERATION}

Permission of this study obtained from Ministry of Health Khartoum state and from Al-neelain University also written consent was tacked from hospital and all participants. Research purpose and objectives was explained to participant in clear simple word. Participants have voluntary to give informed consent. Questionnaire was filled with the participant in their rest time without any interruption to their work.

\section{RESULT}

Table-1: The mean comparison of study parameters in case versus control groups

\begin{tabular}{|l|l|l|l|}
\hline Parameters & Mean \pm SD (Cases) & Mean \pm SD (Control) & P value \\
\hline T.B & $0.91 \pm 0.84$ & $0.63 \pm 0.49$ & 0.107 \\
\hline D.B & $0.17 \pm 0.09$ & $0.18 \pm 0.12$ & 0.644 \\
\hline T.P & $7.36 \pm 1.08$ & $9.99 \pm 5.76$ & 0.271 \\
\hline ALBUMIN & $3.89 \pm 1.01$ & $4.55 \pm 1.05$ & 0.014 \\
\hline ALP & $62.4 \pm 39.9$ & $76.1 \pm 32.5$ & 0.137 \\
\hline AST & $41.4 \pm 33.7$ & $41.2 \pm 20.5$ & 0.975 \\
\hline ALT & $24.8 \pm 12.65$ & $16.8 \pm 9.66$ & 0.016 \\
\hline
\end{tabular}




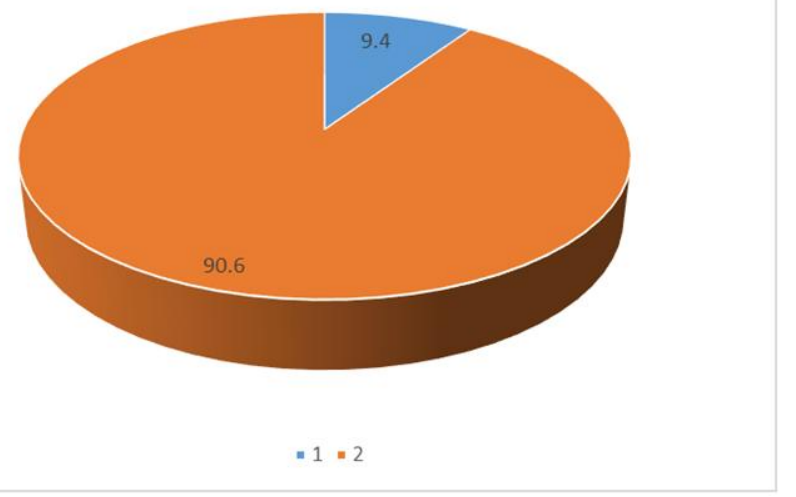

Fig-1: Distribution of study group according to do of drug monitoring test

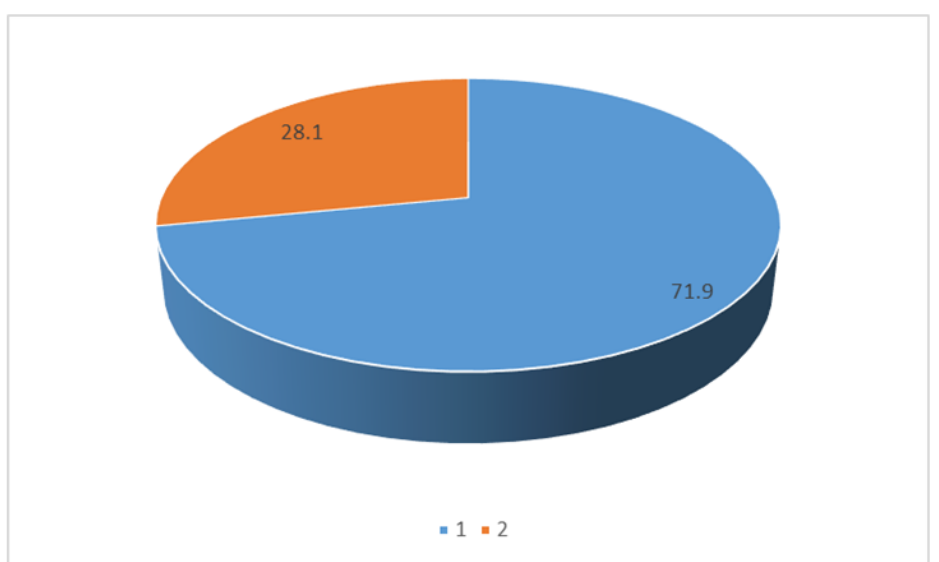

Fig-2: Distribution of study group according to follow to nerology clinic

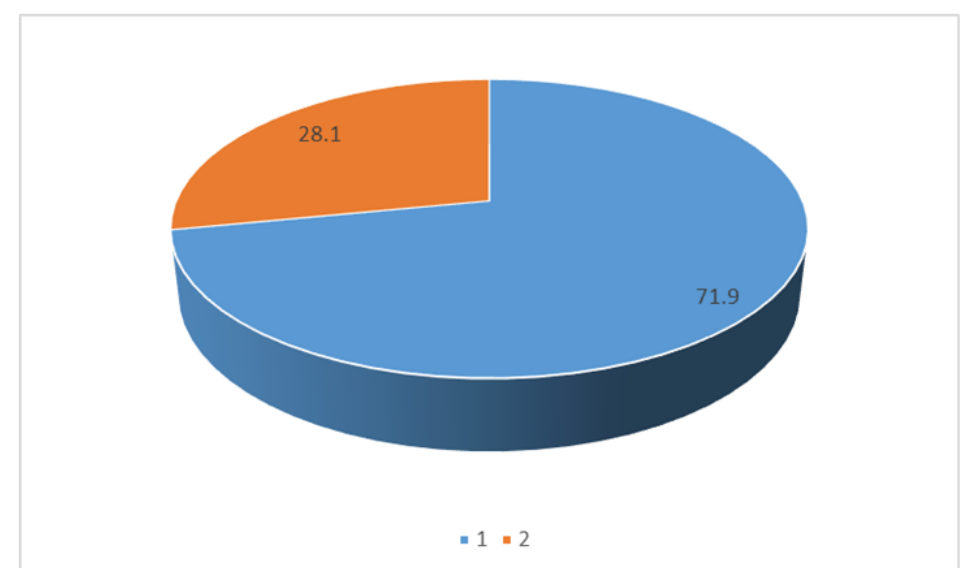

Fig-3: Distribution of study group according to regulation of drug intake

\section{DISCUSSION}

In this case control study a total of 64 epileptic patients were categorized into two groups, group I epileptic patients on antiepileptic drugs for more than one years and group II newly diagnosed epileptic patients and not started antiepileptic drugs. The study revealed that among group (I) there was significant increase in mean serum concentration AST 41.4 \pm 33.7 , ALT 24.8 \pm 12.65 with (p-value 0.975 and 0.016) respectively, compared with mean values of control (II) who reported AST 41.2 \pm 20.5 , ALT 16.8 \pm 9.66 . Moreover, was significant decrease in mean plasma concentration of ALP $(62.4 \pm 39.9)$ in Group (I) compared with mean values in group (II) $(76.1 \pm 32.5)$ (p-value 0.137). Significant decrease in mean plasma albumin concentration $3.89 \pm 1.01$ in group (I) in contrast to group (2) $4.55 \pm 1.05$ (p-value 0.014 ). No significant change in total protein $7.36 \pm 1.08$, total bilirubin $0.91 \pm 0.84$, direct bilirubin $0.17 \pm 0.09$, between the two groups (T.B0.63 \pm 0.49$),(D . B 0.18 \pm 0.12)$. This indicates that antiepileptic drugs had significant effect on changing the levels of liver enzymes and serum proteins in the patients under the study. Our findings similar to what concluded by previous studies. In this regard, Kashinath et al. [8] conducted a study on the 
"effect of phenytoin sodium on liver function tests. They reported that mean value of serum ALP in epileptic patients receiving Phenytoin sodium wass 186.67 which is significantly higher than control group (106.3) $\mathrm{p}<0.001$. The results of the study showed a significant increase in the levels of serum ALP in the epileptic patients on Phenytoin sodium as compared to control group. Dharmesh et al. [9] conducted a study on "liver enzymes activity during sodium valproate therapy on epileptic patients". They found that levels of ALT and AST were significantly increased in patients of epilepsy after administration of Sodium Valproate for 3 months. Aminotransferase levels in Epilepsy patients significantly increased and Pseudocholinesterase levels were significantly decreased, but there were no statistical significant changes in the levels of other liver enzymes like alkaline phosphatase. Ramin et al. [10] concluded that the incidence of increased aminotransferase level in patients on atorvastatin was 18 percent. Ten patients from all of 250 patients (4\%) had more than 3 times increase in liver transferases that the medication was discontinued. Two percent for patients taking less than $80 \mathrm{mg}$ atorvastatin and 5 percent of patients on $80 \mathrm{mg}$ of the drug had more than 3 times elevation in aminotransferase level. Age distribution of the patients was between 40 to 80 years old in $86.6 \%$.

\section{CONCLUSION}

The study revealed that antiepileptic drugs had significant effects in increasing liver enzymes and this necessitate continuous screening of liver function among epileptic patients to overcome the adverse effects of these drugs on liver, and do of drug monatring test is important.

\section{REFERENCES}

1. Fisher, R.S., Acevedo, C., Arzimanoglou, A., Bogacz, A., Cross, J.H., Elger, C.E., Engel, J.Jr., Forsgren, L., French, J.A., Glynn, M., Hesdorffer, D.C., Lee, B.I., Mathern, G.W., Moshé, S.L.,
Perucca, E., Scheffer, I.E., Tomson, T., Watanabe, M., Wiebe, S. (2014). A practical clinical definition of epilepsy. Epilepsia. 2014; 55:475-482.

2. Meinardi, H., Scott, R.A., Reis, R., Saner, J.W.A. (2001). The treatment gap in epilepsy: the current situations and ways forward. Epilepsia, 42(1);136-149.

3. Ngugi, K.A., Bottomley, C., Kleinschmidt, I., Sander, J.W., Newton, C.R. (2015). Estimation of the burden of active and life-time epilepsy: a metaanalytic approach. Epilepsia, 51(5):883-890.

4. Rishe, W., Seifu, M.F., Gelaw, B.K., Gunasekaran, T., Gebremariam, E.T., Mohammed, M.A. (2015). Drug use evaluation of antiepileptic drugs in outpatient epilepsy clinic of Bishoft General Hospital, East Shoa, Ethiopia. Int J Res Dev Pharmacy Life Sci, 4(3):1516-1528.

5. Oka, E., Ohtsuka, Y., Yoshinaga, H., Murakami, N., Kobayashi, K., Ogino, T. (2006). Prevalence of childhood epilepsy and distribution of epileptic syndromes: a population-based survey in Okayama, Japan. Epilepsia, 47(3):626-630.

6. Ahmed, S.N., Siddiqi, Z.A. (2006). Antiepileptic drugs and liver disease. Seizure, 15, 156-164.

7. Björnsson, E. (2008). Hepatotoxicity associated with antiepileptic drugs. Acta Neurol Scand, 118(5):281-90.

8. Kashinath, G., Gajnan, K.P., Padmanabha, T.S. (2014). Effect of Phenytoin Sodium on Liver Function Tests; International Journal of Pharma and Biosciences, 5(1):249-252.

9. Dharmesh, G., Hariom, S., Nitinkumar, C., Nikunj, M., Kalpana, G. (2013). Liver enzymes activity during sodium valproate therapy in patients of epilepsy; International Journal of Research Medicine, 2(2):30-33.

10. Ramin, T.1., Mohammad, B.M. (2015). Evaluation of Atorvastatin Safety on Liver Function Tests, a Prospective Study; Novelty in biomedicine, 3, 99102. 